\title{
Microstructuring Tools for Sheet Bulk Metal Forming - A Designated Application for Picosecond Laser
}

\author{
T. Häfner ${ }^{* 1}$, Y. Reg ${ }^{* 2}$, H. Hetzner ${ }^{* 3}, \underline{\text { M. Schmidt }}{ }^{* 1,2,4}$ \\ ${ }^{*}$ Chair of Photonic Technologies, Friedrich-Alexander University Erlangen-Nuremberg, Germany \\ E-mail: tom.haefner@lpt.uni-erlangen.de \\ *2 Bayerisches Laserzentrum GmbH, Germany \\ ${ }^{* 3}$ Chair of Engineering Design, Friedrich-Alexander University Erlangen-Nuremberg, Germany \\ ${ }^{*}{ }_{4}$ SAOT - Erlangen Graduate School in Advanced Optical Technologies
}

\begin{abstract}
In addition to coatings, microstructures can increase abrasion-resisting quality of sheet bulk metal forming tools by influencing friction conditions. The use of ultrashort pulsed lasers offers a possibility for manufacturing flexible microstructures with very high precision. Thus the processing with picosecond laser allows the generation of structures with dimensions down to the range of single microns. Because of the characteristic ablation process temperature sensitive materials can be structured without any heat transfer into the material. Applying appropriate processing parameters avoids complex post-finishing processes because of the achieved good structure qualities without any melt and any material throw-up. For implemention of a fast structuring process with high ablation rates it is shown, that only fluences near the ablation threshold allow a processing result of high quality and with the maximum ablation efficiency.
\end{abstract}

DOI:10.2961/jlmn.2013.01.0009

Keywords: Ablation efficiency, Ablation quality, Microstructuring, Picosecond laser, Ultrashort pulsed laser

\section{Introduction}

In mechanical forming processes as well as in all areas of industrial production the focus is increasingly lying on saving energy and material resources. Within processes with frictional movements the tribological conditions in the contact zone of the friction partners determine significantly the process boundaries, product quality and wear [1,2].

The occurrence of friction between the components results in local heating of the contact regions, so that mechanical properties change. This material modification e.g. in hardness or in brittleness effects an increased wear of the tool. Thus the reproducibility of the manufacturing process decreases, which ultimately limits the tool life. Therefore tool life is closely associated with the friction conditions.

Coatings consisting of amorphous carbon layers on the tool surfaces offer a possibility for an adaption of the tribological conditions to the strains in the metal forming process $[3,4]$. Another method, which can influence the coefficient of friction locally, can be realized by microstructures on the tool surfaces. Different studies have shown that different friction conditions are adjustable by the shape and the arrangement of the microstructures. This local change of friction values is necessary in sheet bulk metal forming processes because of the strains during the different operations of sheet and bulk metal forming. In sheet metal forming only low surface pressures exist, while bulk metal forming is generally characterized by high surface pressures and surface enlargements [5]. An example for an increase in coefficient of friction is shown in figure 1a. The illustrated crossed arrangement of channels can support the supply of lubricants, but on its own increases the friction value. In contrast, the circular craters shown in figure $1 \mathrm{~b}$ can fulfill a function as oil lubricants and therefore also decrease the friction value.

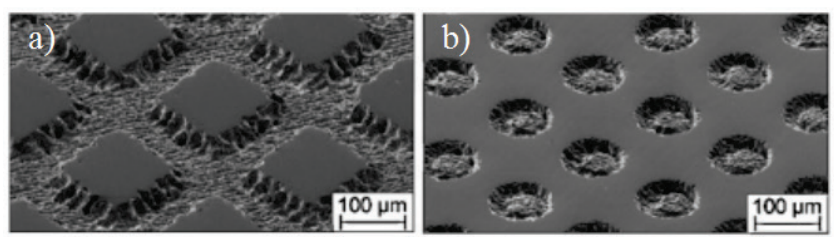

Fig. 1 (a) Crossed channels and (b) craters as an example for microstructures in polished steel surfaces [2]

Those microstructures can be generated by erosive, etching or cutting techniques. Another opportunity, which depends less on material properties such as electrical conduction or hardness, is offered by the use of the laser beam. As previous studies have shown the usage of an excimer laser is a suitable method for structuring almost any material [6].

While the principal suitability of the structuring results, generated for change in friction value has been demonstrated, nevertheless there are some disadvantages of this structuring technique. Due the pulse duration of some nanoseconds the material ablation is caused by a sudden melting, evaporation and plasma formation of the irradiated zone [1] But as a result of the existence of melt during the ablation process material throw-ups at the margin of the microstructures arise. This burr can also have negative effects on friction conditions and therefore has to be removed by an additional processing step.

To overcome this characteristic of short pulse laser ablation the usage of ultrashort pulsed lasers can be useful. 
Due the pulse duration in the range of pico- or femtoseconds the irradiated material will be only influenced in the region of the optical penetration depth, if suited laser parameters are applied. Therefore the meanwhile melt state can be avoided.

Furthermore structuring with ultrashort pulsed lasers offers the possibility to generate structures with dimensions down to the range of single microns. Another advantage in use of ultrashort pulsed lasers is the absence of the mask projection technique, which is used during the structuring with excimer lasers. Thus different structure arrangements can be easily realized by varying the movement path of the laser beam. Also there will not exist friction changing interfaces which can result form a concatenation by the mask covered areas.

This paper focuses on the influence of selected laser parameters on the result of surface treatment with regard to ablation and structure quality, ablation efficiency and processing speed. Correspondingly in this paper the knowledge of generating microstructures is created. Based on this, friction tests with different defined and previously simulated structure sizes and arrangements can be carried out.

\section{Theoretical background}

If material is radiated with an ultrashort laser pulse and the material-depending energy threshold is exceeded, material is removed in the radiated zone and a crater arises. By increasing the pulse energy within the regime above the threshold the crater diameter $D$ rises up. This correlation between the diameter and the pulse energy respectively the peak fluence $\Phi_{0}$ can be described by equation 1 [7], which is based on the Gaussian shaped intensity distribution of a laser beam. Thereby $w_{0}$ defines the beam radius at the beam waist and $\Phi_{t h}$ defines the threshold fluence:

$$
D^{2}=2 * w_{0}^{2 *} \ln \left(\frac{\phi_{0}}{\phi_{t h}}\right)
$$

The dependency of the ablation threshold on the number of pulses $N$ can be described in a model according to Yee [8]. This model explains the decline of the threshold fluence $\Phi_{t h}$ as a result of an increase of $N$. This so-called incubation effect can be quantified by the accumulation factor $S$ according to equation 2, in which $\Phi_{t h, l}$ defines the threshold fluence for one laser pulse:

$$
\phi_{t h}=\phi_{t h, 1} * N^{S-1}(0<S \leq 1)
$$

The ablation process of metals induced by ultrashort laser pulses can be described by the Two-Temperature-Model [9]. The condition for an application of this model is that the timescales of energy coupling between electrons and phonons has to be larger than the pulse duration itself. Hence the typical cold ablation based on photochemical effects induces the material removal [10]. The depth of the crater resulting from this typical ablation is given by the optical penetration depth $\delta$. So that by the knowledge of the crater depth $z_{a b l}$ the optical penetration depth can be determined as a function of increasing peak fluence according to equation 3 [11]:

$$
z_{a b l}=\delta * \ln \left(\frac{\phi_{0}}{\phi_{t h}}\right)
$$

\section{Method of examination}

For the final generation of structure geometries which are suited for influencing friction, the knowledge of the correlation between the laser parameters and the resulting ablation geometry and quality is necessary. For this purpose crater structures were produced by applying selected settings of the process parameters peak fluence $\Phi_{0}$ and number of pulses $N$. Additionally the possibility of scaling the process speed by increasing the pulse frequency $f_{P}$ was verified under observation of a change in ablation quality and efficiency.

\subsection{Material and sample treatment}

The used target material was tool steel 1.3343, which is a so-called high-speed steel and contains the listed chemical elements according to [12], as shown in table 1 . The polished surfaces of the samples which are available in the shape of discs with $30 \mathrm{~mm}$ in diameter have an initial roughness of $R a=0,020 \mu \mathrm{m}$.

Table 1 Chemical composition of steel 1.3343 [12]

\begin{tabular}{cccccc}
\hline Element & C & Cr & Mo & V & W \\
\hline Share in weight percent & 0,9 & 4 & 5 & 1,9 & 6,4
\end{tabular}

Before laser processing the sample surface was cleaned with acetone to remove any lubricant residues and thus to enable constant absorption conditions. For an evaluation after laser structuring a cleaning step in an ultrasonic bath is carried out to remove any ablated particles and debris.

\subsection{Experimental setup}

The ultrafast laser used for work was the picosecond laser system Fuego ${ }^{\mathrm{TM}}$ of the company Time-Bandwidth. This laser system provides a maximum average power of $50 \mathrm{~W}$ and $25 \mathrm{~W}$ at a maximum pulse frequency of $f_{P}=8.2 \mathrm{MHz}$ and the used wavelengths of $1064 \mathrm{~nm}$ or $532 \mathrm{~nm}$. By using the pulse picker function the pulse frequency can be reduced down to the radiation of single pulses. The basic experiments were done with a pulse frequency $f_{P}=50 \mathrm{~Hz}$ to avoid any heat accumulation by leading laser pulses. The optical setup contains each two beam expanders within the beam paths for both wavelengths. The movement of the laser beam on the sample at the infrared wavelength is carried out by means of a Galvoscanner system hurryScanII. The existing spot diameter after focusing by an $\mathrm{f}$-theta lens with the focal length $f=160 \mathrm{~mm}$ amounts to $2 *_{w_{0}} \approx 31 \mu \mathrm{m}$. In contrast, the optical setup for the visible wavelength is realized with a sample movement by means of an axis system from Aerotech. The expanded raw beam is focused with a lens of $f=100 \mathrm{~mm}$ focal length to the spot diameter $2 * w_{0} \approx 35 \mu \mathrm{m}$. Every experimental condition has the sample size $n=3$, as it is shown in the graphs.

\subsection{Analysis methods}

The surface topography was recorded by means of a laser scanning microscope (LSM), so that the diameter and depth of the craters can be measured. The evaluation of the structure quality was implemented by using images generated with a light microscope and a scanning electron microscope (SEM). 


\section{Results and discussion}

\subsection{Ablation threshold and penetration depth as a function of the wavelength and the number of pulses}

The dependency of the mean ablation threshold on the number of pulses has been examined for $1064 \mathrm{~nm}$ and $532 \mathrm{~nm}$ wavelength. The mean threshold values have been determined by using equation 1 . As it is visible in figure 2 the mean threshold fluence decreases with increasing number of pulses. The reason for this correlation can be justified by the incubation effect.

The material surface layer is changed by ablation and melt processes which are induced by the energy coupling. On the one hand the surface topography changes, on the other hand defects within the layer are induced. Sometimes also an oxidation of the surface occurs. This surface modifications contribute to an increase in absorption and accordingly to a decrease of the ablation threshold [8].

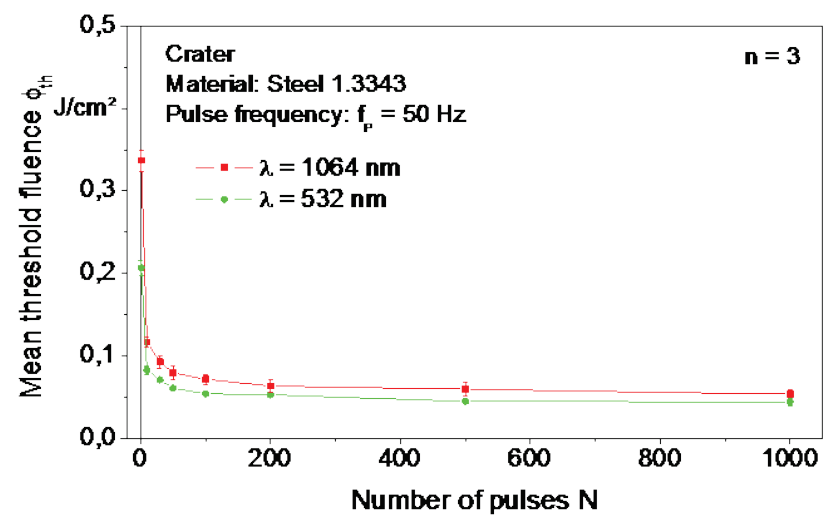

Fig. 2 Pulse number-dependent mean ablation thresholds at $\lambda=1064 \mathrm{~nm}$ and $\lambda=532 \mathrm{~nm}$

This decrease in threshold fluence can be described by the incubation factor $S$, which has been determined to be $S=$ $0.69 \pm 0.04$ for $\lambda=1064 \mathrm{~nm}$, according to equation 2 . This value indicates a high dependence of the ablation threshold of the number of pulses. This is mainly attributed due to the initial state of the polished sample surface with its roughness $R a=20 \mathrm{~nm}$. This roughness is strongly increased by previous laser pulses. For the wavelength $\lambda=532 \mathrm{~nm}$ the factor $S=0.81 \pm 0.03$ arises analogous. Both calculated values agree well with the usual values for metals $[11,13,14]$.

Additionally these surface modifications are detectable by a slight decrease in the optical penetration depth $\delta$ with an increasing number of pulses $N$. The values for the number of pulses $N=50$, which are calculated from the crater depth corresponding to equation 3 , are $\delta=7.9 \mathrm{~nm}$ at $\lambda=1064 \mathrm{~nm}$ and $\delta=6.2 \mathrm{~nm}$ at $\lambda=532 \mathrm{~nm}$. For the tenfold number of pulses $N=500$ the optical penetration depths amount to $\delta=6.3 \mathrm{~nm}$ respectively to $\delta=5.2 \mathrm{~nm}$.

For a comparison of the wavelength-dependent experimentally determined values of the ablation thresholds, see table 2 , it is distinctive that for the microstructuring at the visible wavelength the material removal starts at lower fluences. One reason for this lower ablation threshold of the tool steel is the absorption at $\lambda=532 \mathrm{~nm}$, which is $33 \%$ higher than at $\lambda=1064 \mathrm{~nm}$. Furthermore the optical pene- tration depth for this visible wavelength is about $20 \%$ smaller, so that the energy coupling of the photons towards the electrons occurs in a smaller region near the surface.

Table 2 Ablation thresholds for selected numbers of pulses

\begin{tabular}{ccccc}
\hline & $\Phi_{t h, 1064}$ & $S-\Phi_{t h, 1064}$ & $\Phi_{t h, 532}$ & $S-\Phi_{t h, 532}$ \\
\hline $\boldsymbol{N}=\mathbf{1}$ & $0.337 \mathrm{~J} / \mathrm{cm}^{2}$ & $\pm 0.013 \mathrm{~J} / \mathrm{cm}^{2}$ & $0.207 \mathrm{~J} / \mathrm{cm}^{2} \pm 0.009 \mathrm{~J} / \mathrm{cm}^{2}$ \\
$\boldsymbol{N}=\mathbf{1 0}$ & $0.117 \mathrm{~J} / \mathrm{cm}^{2}$ & $\pm 0.007 \mathrm{~J} / \mathrm{cm}^{2}$ & $0.083 \mathrm{~J} / \mathrm{cm}^{2} \pm 0.006 \mathrm{~J} / \mathrm{cm}^{2}$ \\
$\boldsymbol{N}=\mathbf{1 0 0}$ & $0.072 \mathrm{~J} / \mathrm{cm}^{2}$ & $\pm 0.006 \mathrm{~J} / \mathrm{cm}^{2}$ & $0.055 \mathrm{~J} / \mathrm{cm}^{2} \pm 0.002 \mathrm{~J} / \mathrm{cm}^{2}$ \\
$\boldsymbol{N}=\mathbf{1 0 0 0}$ & $0.054 \mathrm{~J} / \mathrm{cm}^{2}$ & $\pm 0.005 \mathrm{~J} / \mathrm{cm}^{2}$ & $0.045 \mathrm{~J} / \mathrm{cm}^{2} \pm 0.005 \mathrm{~J} / \mathrm{cm}^{2}$
\end{tabular}

But due to the approximately $20 \%$ lower optical penetration depth of the tool steel at $\lambda=532 \mathrm{~nm}$ the maximum energy amount, which is necessary for an exclusively cold removal, is less than at $\lambda=1064 \mathrm{~nm}$. Accordingly, also the maximum ablation rate for a cold material ablation will be actually lower in the visible wavelength range than in NIR. Because of the lower maximum mean laser power, based on the frequency conversion, the potential for reduction of processing time at $\lambda=532 \mathrm{~nm}$ is smaller than at $\lambda=1064 \mathrm{~nm}$. Due to these two facts the following detailed investigation of influencing the structure results have been carried out only in NIR.

\subsection{Structure quality depending on the peak fluence and the number of pulses}

In this section the ablation quality with regard to the impact of an increase in peak fluence will be described. For this purpose, the formation of melt on the structure ground and the margins will be observed.

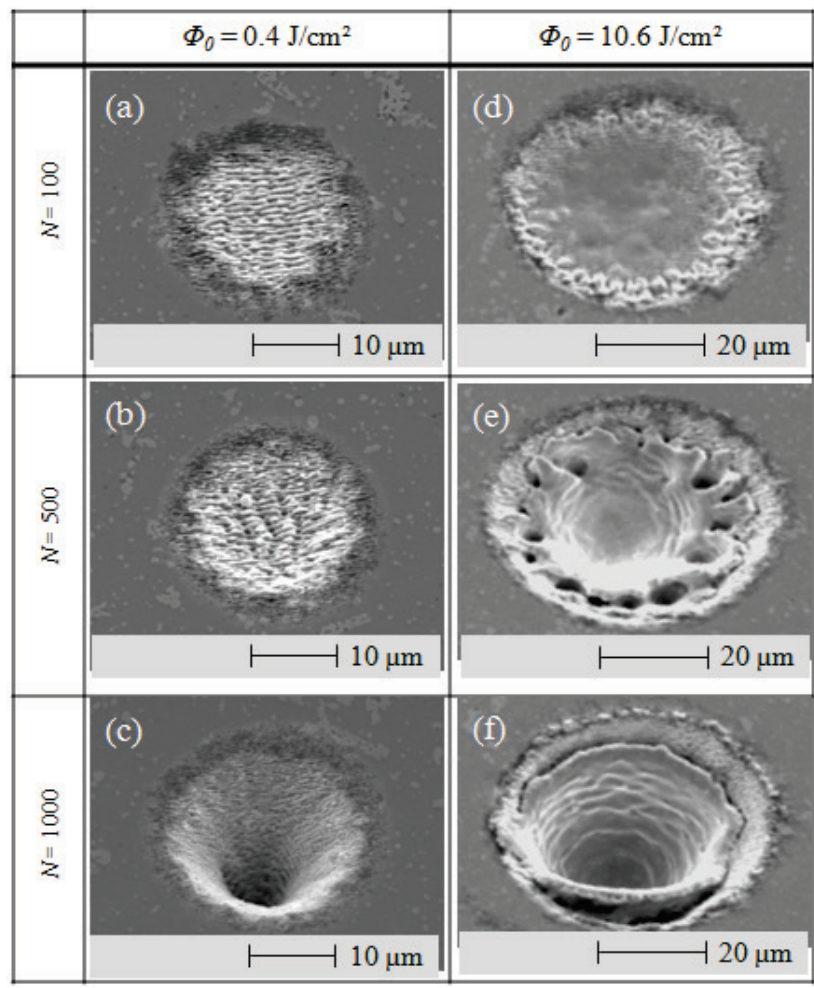

Fig. 3 SEM images of the ablated craters for justifying the structure quality $\left(\lambda=1064 \mathrm{~nm} ; f_{P}=50 \mathrm{~Hz}\right)$

The material removal with peak fluences in an energy regime just above the ablation threshold, as it is labelled and 
named with (I) in figure 4, is characterized by an absence of melt. Thus, there are neither material throw-ups at the margins nor melt residues within the crater, as it is shown in figure 3a. By using linear polarized laser radiation laserinduced periodic surface structures (LIPSS) arise, which are typical for metals [15]. In this fluence regime these LIPSS determine the roughness of the structure ground. Specifically, the height and the periodicity of those ripples, which are pronounced perpendicular to the polarization direction of the incident laser radiation, amount less than $300 \mathrm{~nm}$ at a pulse number $N=100$.

Deeper craters can be generated by increasing the number of pulses. In this case the roughness of the structure ground rises up slightly. This phenomenon is caused by the formation of additional LIPSS, which extend perpendicular to the before mentioned ripples, as seen in figure $3 \mathrm{~b}$. With a further increase of the crater depth the size of those regular sub-structures declines, see figure $3 \mathrm{c}$.

If the peak fluence is increased, higher ablation rates can be achieved. In this fluence regime, labeled with (II) in figure 4, the formation of a melt film at the structure ground occurs already at low pulse numbers $N$, as shown in figure $3 \mathrm{~d}$. Accordingly, an irregular surface with a higher roughness is forming. The irregularities expand during a rise of number of pulses, see figure $3 \mathrm{e}$. These large irregularities can result in local higher crater areas, which are even expanded above the initial sample surface. Also at high pulse numbers a part of the occurring melt solidifies at the crater edges as throw-ups, see figure $3 \mathrm{f}$.

As a result, this fluence regime (II) is not suited for a generation of friction reducing microstructures. Thus, from these oberservations we can first derive that only low peak fluences can be used for structuring craters with low roughness and absence of material throw-ups.

\subsection{Influence of the fluence on the ablation efficiency}

The major reason for the decreasing structure quality of the ablated crater can be named by the formation of melt, as observed by the SEM images.

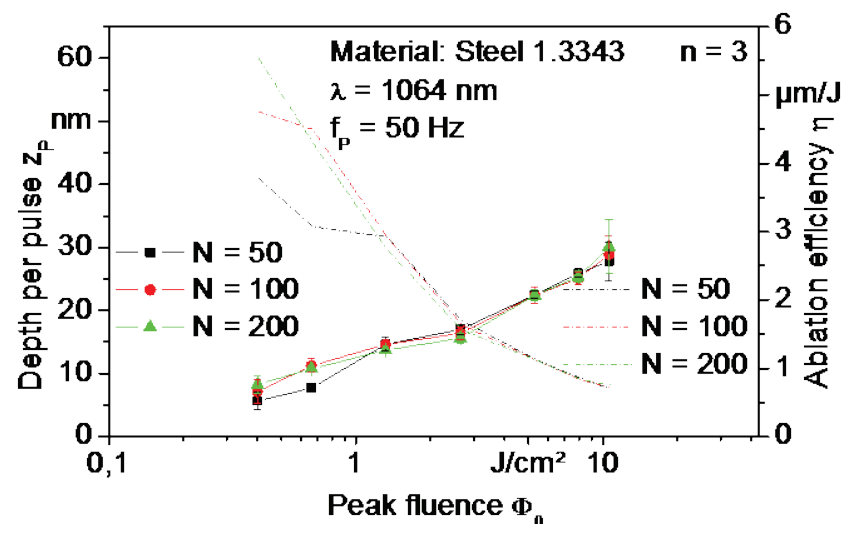

Fig. 4 Influence of an increasing peak fluence on depth per pulse and ablation efficiency

But before the melt is visibly deposited on the material surface a heat influence of the radiated area and sometimes surrounding regions depending on the amount of coupled energy happens. This heat transfer to the parental material can be detected by a decline of the energetic ablation effi- ciency, which is presented at the axis of ordinates on the right side of the graph in figure 4.

It is seen that the energetic ablation efficiency falls continuously with increasing peak fluence. Though there is a peak fluence, where a maximum ablation efficiency can be reached. As Schmid described, this optimal fluence is proportional to the ablation threshold, according to equation 4 , and to the optical penetration depth $\delta[16]$ :

$$
\phi_{0, o p t}=\frac{e^{2}}{2} * \phi_{t h}
$$

With regard to the ablation threshold for $N=100$, as listed in table 2 , this optimal peak fluence can be calculated to $\Phi_{0, \text { opt }}=0.27 \mathrm{~J} / \mathrm{cm}^{2}$. So that, this optimal peak fluence is not covered by the considered peak fluences. Therefore only the decrease of ablation efficiency can be seen in figure 4. The optimal value of the fluence can be physically interpreted so far, that the incident energy of the laser pulse is completely absorbed within the optical penetration depth. Thus, only the area of optical penetration depth is affected by the coupled energy and eventually completely ablated.

The impact of this physical effect is additionally visualized by figure 5. Applying the same total peak fluence $\Phi_{0, \text { tot }}$ different crater depths can be generated. This cumulative amount of energy can be calculated by $\Phi_{0, t o t}=N * \Phi_{0}$. As seen in figure 5, always the constellation with lower peak fluence and therefore the higher number of pulses leads to the formation of a structure with a larger crater depth $z_{a b l}$.

The better ablation efficiency at lower peak fluences also causes this observation. Furthermore we can conclude that a desired rise of the crater depth will be easily realized by increasing the number of pulses, if the peak fluence has to be constant.

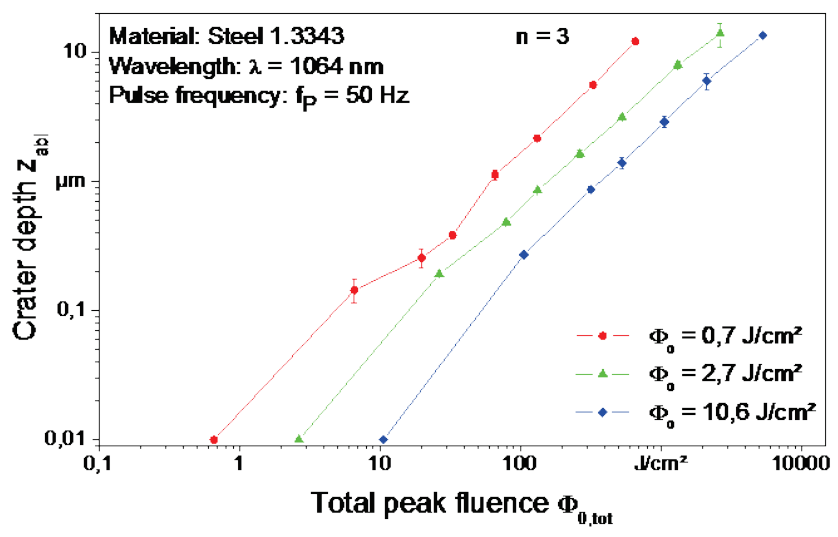

Fig. 5 Correlation between crater depth and total peak fluences

Thus, for possible highest energy-efficient ablation fluences slightly above the ablation threshold are relevant for microstructuring. Specifically the selected fluence should not exceed the tenfold value of the ablation threshold. As a result for processing within this lower energy regime the ablation rate is very low. But for an industrial application the required high removal rates and thus short process times have to be implemented. Therefore the pulse frequency must be increased far beyond the investigated $f_{P}=50 \mathrm{~Hz}$. 


\subsection{Influence of the pulse frequency on structure quali- ty and ablation efficiency}

Accordingly to the findings described before, an investigation of the ablation behavior as a result of a change of the pulse frequency is necessary for a final acceleration of the structuring process. For this purpose a fluctuation in crater cross-sectional area $A_{a b l}$ as a function of the pulse frequency $f_{P}$ was investigated. The peak fluence and the number of pulses were kept constant as well. First, it is visible by figure 6 that the structure quality remains approximately the same. This means, that the roughness of the structure ground is set due to the arised LIPSS again. For a comparison of the crater geometry including diameter and depth, it is visible that the diameter slightly decreases by a growth of the pulse frequency, as seen in figure $6 \mathrm{~b}$. Also the measured crater cross-sectional area decreases significantly.

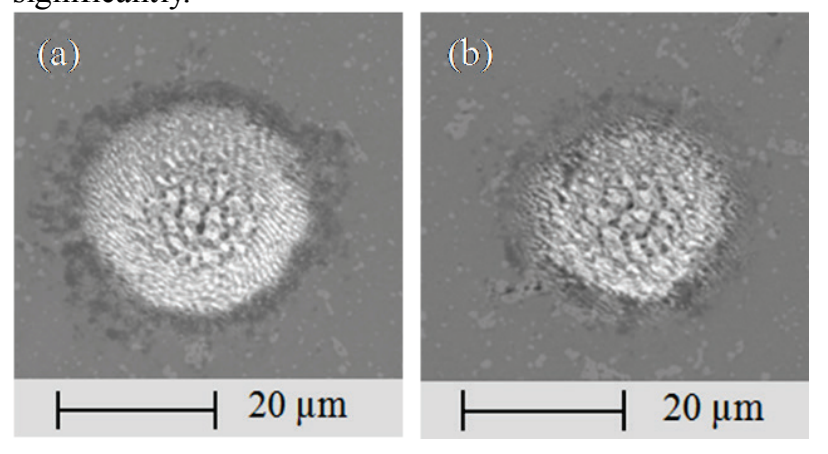

Fig. 6 SEM images of craters with the same structure quality generated with the following parameters: $\Phi_{0}=0.4 \mathrm{~J} / \mathrm{cm}^{2} ; \mathrm{N}=500$ and

(a) $f_{P}=200 \mathrm{kHz}$ respectively (b) $f_{P}=1000 \mathrm{kHz}$

Observing the crater cross-sectional area as a function of the pulse frequency this drop is also visible at higher fluence, as seen in figure 7. This decline in ablation efficiency is caused by plasma shielding, which implies that the trailing laser pulse is partly reflected and scattered by the ablated particles in the plasma of the leading pulse [17]. Therefore less energy of the laser beam hits the surface and can be coupled to the material, so that the trailing pulses are influenced by ablated material. The efficiency drop is significant at the higher peak fluence $\Phi_{0, o p t}=2.7 \mathrm{~J} / \mathrm{cm}^{2}$ as the induced plasma has an higher density due to the higher pulse energy.

Already at lower pulse frequency a slight rise of ablation efficiency occurs. This increase towards $200 \mathrm{kHz}$ respectively $50 \mathrm{kHz}$ can be attributed to the heat accumulation. In addition, the determination of the crater dimension is fraught with a higher measurement uncertainty because of the described melt formation at higher peak fluences.

With reference to the almost horizontal graph for the lower fluence it can be seen that a scaling of the structuring process for crater generation is possible. As seen in figure 6 the structure quality of the ablated craters at pulse frequencies of $f_{P}=0.05 \mathrm{kHz}, f_{P}=200 \mathrm{kHz}$ and $f_{P}=1 \mathrm{MHz}$ are quite similar and also sufficient, because they do not have any irregularities. Thus, the processing time for structuring tool steel can be reduced by a factor of 20 thousand with only a significant loss in ablation efficiency for the relevant low peak fluences.

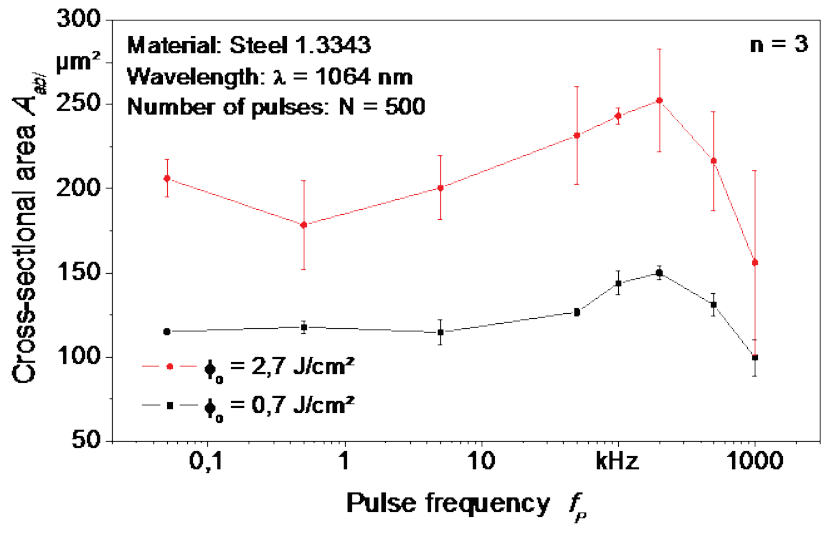

Fig. 7 Dependency of the crater cross-sectional area on the pulse frequency and peak fluence

\section{Summary and outlook}

It can be concluded, that microstructures in the shape of craters with a high structure quality can be generated by using the picosecond laser with a wavelength at $\lambda=1064 \mathrm{~nm}$ and also at $\lambda=532 \mathrm{~nm}$. The optical penetration depths at these wavelengths are quite short. Because of a sligthly longer penetration depth at $\lambda=1064 \mathrm{~nm}$ bigger craters can be ablated without thermal influence of the surrounding material compared to $\lambda=532 \mathrm{~nm}$. However, only a limited energy regime is useful for a material removal without melt formation and consequently material throwups. This energy regime for cold ablation is slightly wider in NIR than in VIS due the longer optical penetration depth. Thus higher ablation rates are possible at $\lambda=1064 \mathrm{~nm}$.

Therefore only fluences below the tenfold value of the ablation threshold are suited for producing structures with a sufficiently smooth bottom. Because of the achieved structure qualities no post processing is necessary.

Applying these low fluences allows structuring with sufficient quality while reaching maximum ablation efficiency. Because of the resulting low ablation rates and long structuring process an increase in pulse frequency is necessary. As it is shown within this paper, a significant change in the ablation efficiency occurs with higher pulse frequency. Therefore a scaling of the ablation process is not simply possible. Despite differing structure geometries for selected pulse frequencies the same structure qualities can be achieved at low peak fluences. These parameters are suited for generating defined crater geometries and selected structure arrangements to quantify friction change on forming tools.

\section{Acknowledgments}

The authors gratefully acknowledge the Deutsche Forschungsgemeinschaft (DFG) for funding the work presented within the sub-project B4 of the SFB / TR 73 "sheet metal forming".

\section{References}

[1] M. Geiger; U. Popp; U. Engel: Excimer Laser Micro Texturing of Cold Forging Tool Surfaces - Influence on Tool Life. In: CIRP Annals - Manufacturing Technology, Vol. 51 (2002), S. 231-234

[2] K.-H. zum Gahr; R. Wahl; K. Wauthier: Experimental Study of the Effect of Microtexturing on Oil Lubricat- 
ed Ceramic/Steel Friction Pairs. In: Wear, Vol. 267 (2009). S. 1241-1251

[3] E. Doege; B. Behrens: Handbuch Umformtechnik. 2. Auflage. Heidelberg: Springer Verlag, 2010

[4] J. Koskinen, et al.: Friction Reduction by Texturing of DLC Coating Sliding Against Steel under Oil Lubrication. In: Surface \& Coatings Technology, Vol. 204 (2010), S. 3794-3797

[5] H.U. Vierzigmann; M. Merklein; U. Engel: Friction Conditions in Sheet-Bulk Metal Forming. In: Procedia Engineering, Vol. 19 (2011), S. 377-382

[6] U. Popp: Grundlegende Untersuchungen zum Laserstrahlstrukturieren von Kaltmassivumformwerkzeugen. Dissertation. Universität Erlangen-Nürnberg, Lehrstuhl für Fertigungstechnologie, 2006

[7] J.M. Liu: Simple technique for measurements of pulsed gaussian-beam spot sizes. In: Optics Letters 7, 5 (1982). S. $196-198$

[8] Y. Jee; M.F. Becker; R.M. Walser: Laser-induced damage on single-crystal metal surfaces. In: Journal of the Optical Society of America B, Vol 5 (1988). S. 648 $-659$

[9] B.N. Chichkov; C. Momma; S. Nolte; F. von Alvensleben; A. Tünnermann: Femtosecond, picosecond and nanosecond laser ablation of solids. In: Applied Physics A, 63 (1996). S. 109 - 115

[10] Y. Reg; C. Kägeler; M. Schmidt: Experimental Studies of Effects at Micro-structuring of Highly Reflecting
Metals using Nano- and Picosecond-lasers. In: Physics Procedia, Vol. 5 (2010), S. 245-253

[11]B. Jaeggi; B. Neuenschwander; M. Schmid; M. Muralt; J. Zuercher; U. Hunziker: Influence of the Pulse Duration in the ps-Regime on the Ablation Efficiency of Metals. In: Physics Procedia, Vol. 12 (2011), S. 164-171

[12]DIN EN ISO 4957 : 1999, Werkzeugstähle

[13]P.T. Mannion; et al.: The effect of damage accumulation behaviour on ablation threshold and damage morphology in ultrafast laser micro-machining of common metals in air. In: Applied Surface Science, Vol. 233 (2004). S. $275-287$

[14] G. Raciukaitis; M. Brikas; M. Gedvilas: Efficiency aspects in processing of metals with high-repetitionrate ultra-short-pulse lasers.

[15] V.S. Mitko et al.: Properties of High-Frequency SubWavelength Ripples on Stainless Steel 304L under U1tra Short Pulse Laser Irradiation. In: Physics Procedia, Vol. 12 (2011), S. 99-104

[16] M. Schmid; B. Neuenschwander; V. Romano; B. Jaeggi; U. Hunziker: Processing of metals with pslaser pulses in the range between 10 ps and 100ps. Proceedings of SPIE - The International Society for Optical Engineering, 7920, 2011

[17] A. Ancona; S. Döring; C. Jauregui et al.: Femtosecond and picosecond laser drilling of metals at high repetition rates and average powers. In: Opt. Lett. Vol. 34, Nr. 21 (2009). S. 3304

(Received: June 15, 2012, Accepted: November 27, 2012) 